\title{
Transduction of a Proteus vulgaris Strain by a Proteus mirabilis Bacteriophage
}

\author{
By J. N. COETZEE \\ Department of Microbiology, University of Pretoria, South Africa
}

(Received 20 January 1975; revised 4 March 1975)

SUMMARY

Only Proteus vulgaris strain PVI27 out of many $P$. vulgaris, $P$. morganii and Providence strains was transduced to kanamycin resistance by high-frequency transducing variants, 5006MHFT $k$ and $5006 \mathrm{MHFT} a k$, of phage $5006 \mathrm{M}$, a general transducing phage for $P$. mirabilis strain PM5006. The phages adsorbed poorly to strain PVI 27 and did not form plaques. The transduction frequency of PVI 27 by these phages was $5 \times 10^{-8} /$ p.f.u. adsorbed. Phage $5006 \mathrm{M}$ increased the transduction frequencies. Abortive transductants were not detected. Transductants segregated kanamycin-sensitive clones at high frequency and this, together with data from the inactivation of transducing activity of lysates by ultraviolet irradiation, indicated that transduction was by lysogenization. The general transducing property of the phages was not expressed in transductions to auxotrophs of PVI 27. Transductants (type I) resulting from low multiplicities of phage input adsorbed phage to the same extent as PVI 27. This suggested a defect in the transducing particles (or host) because single phage 5006M infection converted strain PM5006 to non-adsorption of homologous phage. Type I transductants did not liberate phage, suggesting a defective phage maturation function. Transductants (type II) which arose from higher multiplicities of phage input did not adsorb phage, indicating possible heterogeneity among transducing particles. Phage derived from type II transductants adsorbed poorly to PVI27 and transduced it to kanamycin resistance at frequencies similar to those of phages $5006 \mathrm{MHFT} k$ and $5006 \mathrm{MHFT} a k$, ruling out host-controlled modification as a cause of the low transduction frequencies. This phage transduced PM5006 to antibiotic resistance at high frequencies but generalized transduction was again not detected. It was suggested that general transduction could be performed by particles which, due to a different composition and/or mode of chromosomal integration, made material they carried susceptible to host-cell modification.

\section{INTRODUCTION}

Proteus mirabilis, $P$. vulgaris, $P$. morganii, $P$. rettgeri and Providence strains may be readily distinguished by simple biochemical tests (Carpenter, 1964). They do however have properties in common and their arrangement is confused (Coetzee, 1972). With the exception of $P$. morganii, strains of the remainder have similar guanine plus cystosine molar percentages (Falkow, Ryman \& Washington, 1962). Although DNA from strains of $P$. vulgaris binds $90 \%$ of $P$. mirabilis nucleotide sequences, the latter share only about $15 \%$ of sequences with Providence and $5 \%$ with P. rettgeri (Brenner \& Falkow, 197I). Members of the Proteus group (Coetzee, 1972) have some phage receptors in common (Coetzee, $1963 a, b, c)$ and host-controlled modification systems have also been described (Coetzee \& Smit, 1969, 1970). However, generalized transducing phages for each of the members of the group (see Coetzee, 1972) showed strict strain specificity, with the exception of the 
Table I. Bacteria, plasmids and bacteriophages

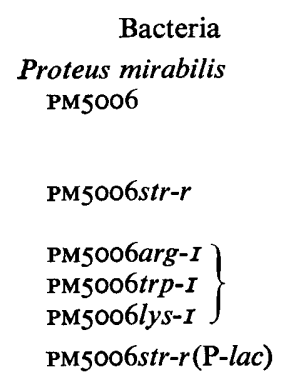

PM5006(P-lac $\overline{\mathrm{R} 447 \mathrm{~b}})$
$P$. vulgaris I 7 strains, including strain PVI27

\section{$P$. morganii 60 strains \\ Providence IOO strains}

\section{Escherichia coli J62-I}

\section{Bacteriophages 5006MHFTk}

5006MHFTak

$5006 \mathrm{M}$

5006M-PM5006str-r 5006M·PM5006arg-I 5006M-PM5006lys-I 5006M-PM5006trp-I
Properties

Host for phages used. Cryptically lysogenic for phage 5006M

Spontaneous mutant resistant to I $\mathrm{mg}$ streptomycin $/ \mathrm{ml}$

Auxotrophs of PM5006

PM5006str-r with P-lac introduced by conjugation. The plasmid transfers the lac marker to other Proteus and Escherichia coli spp. at frequencies of about $10^{-1} /$ donor cell

PM5006 with the fused plasmid P-lac $\overline{\mathrm{R} 447 \mathrm{~b}}$ introduced by conjugation. The plasmid transfers markers lac and kanamycin resistance to other Proteus spp. and E. coli $\mathrm{J} 62-\mathrm{I}$ at frequencies of about $10^{-1} /$ donor cell

Typical $\boldsymbol{P}$. vulgaris strains; 30 sensitive to ampicillin and kanamycin, 87 including PVI27 resistant to ampicillin and sensitive to kanamycin

Typical $P$. morganii strains. Resistant to ampicillin, sensitive to kanamycin

44 strains of subgroup A. Resistant to ampicillin, sensitive to kanamycin 56 strains of subgroup B. 40 resistant to kanamycin, sensitive to ampicillin. I6 resistant to ampicillin, sensitive to kanamycin

$\mathbf{F}^{-}$lac, pro, his, trp, nal-r mutant of E. coli $\mathrm{K} \mathrm{I} 2$

Lysate from u.v.-induced PM5006( $\overline{(\mathrm{R} 394})^{*}$. High-frequency transducing for kanamycin resistance

Lysate from u.v.-induced PM5006 $\left(\overline{\overline{\mathbf{R}_{394}}}\right) \dagger$. High-frequency transducing for ampicillin and kanamycin resistance

Cryptic prophage of PM5006. Produced by lytic infection of PM5006. Generalized transducing for PM5006. Serologically identical to phages $34,5006 \mathrm{MHFT} k$ and 5006MHFTak

Phage 5006M lysate of PM5006str-r

Phage 5006M lysate of PM5006arg-I

Phage 5006M lysate of PM5006lys-I

Phage 5006M lysate of PM5006trp-I
Reference or source

Coetzee \& Smit (I970)

Krizsanovich (1973)

Coetzee (1974 $b, 1975)$

Coetzee (I974b)

Coetzee $(1974 b)$

Coetzee (1974a)

Falkow et al. (1964)

Coetzee (1974a)

Hedges et al. (1973)

Coetzee (unpublished)

Carpenter (1964)

Dr H. C. de Klerk

Dr H. J. Koornhof

Carpenter (1964)

Carpenter (1964)

Dr H. C. de Klerk

Dr H. J. Koornhof

Clowes \& Hayes (1968)

Coetzee, Datta \& Hedges (1972)

Coetzee (1974b)

Coetzee (1975)

Krizsanovich (1973)

Coetzee \& Sacks (1960)

Coetzee (1974 $b, 1975)$

* Superior line indicates that the plasmid was transduced to PM5006. The T compatibility R factor R394 was isolated from a strain of $P$. rettgeri and carries markers for resistance to ampicillin and kanamycin. The $\mathbf{R}$ factor was transferred to PMI 3 by conjugation. Phage 34 lysates of the ampicillin- and kanamycinresistant progeny were used to transduce the markers to PM5006. Some transductants only expressed the kanamycin-resistance marker, and u.v. induction of these transductants yielded high-frequency-transducing phage lysates for kanamycin resistance.

$\dagger$ Superior lines indicate that the plasmid was transduced to a derivative of PM5006 $(\overline{\mathrm{R} 394})$. 
$P$. mirabilis phages 34 and I3vir (Coetzee \& Smit, 1969, 1970) and Providence transducing phages (Coetzee, Smit \& Prozesky, 1966) which were active on a few other strains of $P$. mirabilis and Providence respectively.

The isolation from one of the $P$. mirabilis transducing phages of variants capable of highfrequency transduction (HFT) of antibiotic resistance markers (Coetzee, 1974 b, 1975) provided a means of selection for possible transductants from strains of Proteus normally not sensitive to these phages. Selection of this kind has been employed by Kondo \& Mitsuhashi (1966) and Goldberg, Bender \& Streicher (1974) (see also Gottesman, Hicks \& Gellert, 1973) to extend the host range of Escherichia coli phage PI to some other genera of the family Enterobacteriaceae. Encouraged by these results it was decided to test the $P$. mirabilis HFT phages on a number of $P$. vulgaris, $P$. morganii and Providence strains.

\section{METHODS}

Bacteria, plasmids and phages. These are presented in Table I. All strains sensitive to kanamycin were tested with both 5006MHFT $k$ and 5006MHFT $a k$ phages for transduction to kanamycin resistance. Strains which were ampicillin-sensitive were tested for transduction to ampicillin resistance by phage 5006MHFT $a k$. Incubation temperature was $37^{\circ} \mathrm{C}$.

General phage techniques. These were according to Adams (1956) and Coetzee (1974b). Freeze-dried phage 34 antiserum (Coetzee \& Sacks, 1960) with a neutralization constant $(K)$ of $180 \mathrm{~min}^{-1}$ against phages 5006MHFT $k$ and 5006MHFTak was used.

Media and antibiotics. These were according to Coetzee (1974b).

Phage adsorption. The method of Kondo \& Mitsuhashi (I966) was used. Phage (0.I ml) was added to $\mathrm{I} \mathrm{ml}$ of a culture of a streptomycin-sensitive test strain in the exponential phase of growth (about $6 \times \mathrm{IO}^{8} / \mathrm{ml}$ ) at a multiplicity of input (m.i.) of plaque-forming units (p.f.u.) of about 2. At o min and after 20 min incubation samples were diluted in ice-cold broth and titrated on PM5006str- $r$ using media which contained streptomycin. Some tests were done with stationary-phase organisms, and adsorption temperatures of 25 and $30^{\circ} \mathrm{C}$ were also investigated.

Transduction. The Millipore membrane filter technique was used (Coetzee, 1974b). Possible hosts were screened at a m.i. of p.f.u. of about 10 , with adsorption for $40 \mathrm{~min}$. In other experiments adsorption was reduced to $15 \mathrm{~min}$. This was done to try and ensure individuality of infected bacteria impinged on the membranes. The phage $\mathrm{m}$.i. in these experiments varied as indicated.

Selection of antibiotic-sensitive segregants. Suitable dilutions of overnight broth cultures of transductants were plated on MacConkey agar. After overnight incubation antibioticsensitive clones were detected by replication on to antibiotic-containing agar. All transductant and segregant clones studied were purified by streaking on the same agar. A colony was then suspended in phage 34 antiserum and incubated for $\mathrm{I} h$ before re-streaking for single colonies.

Ultraviolet irradiation of phage lysates. Ultraviolet (u.v.) irradiation was by the method of Coetzee (1974b).

Selection of auxotrophic or ampicillin-sensitive mutants and phage-sensitive mutants. The $N$-methyl- $N^{\prime}$-nitro- $N$-nitrosoguanidine method of Adelberg, Mandel \& Chen (I965) was used. Suitable dilutions of treated cultures were plated on MacConkey agar and incubated overnight. Ampicillin-sensitive clones were screened for by replication on to agar containing the antibiotic, while auxotrophs were detected by replication on to minimal medium. Phage was also spotted on lawns of 5000 treated colonies. 
Table 2. Transduction of P.vulgaris PVI27 and P. mirabilis PM5006 by phage 5006MHFTk

Transductions were done as outlined in Methods. Adsorption was for $20 \mathrm{~min}$, and for kanamycin resistance, membranes were incubated on nutrient agar for $\mathrm{I} h$ before transfer to MacConkey agar containing $50 \mu \mathrm{g} \mathrm{kanamycin} / \mathrm{ml}$. For detection of prototrophs, membranes were placed on minimal medium directly after filtration.

\begin{tabular}{|c|c|c|c|c|c|}
\hline \multirow{2}{*}{$\begin{array}{c}\text { Experiment } \\
\text { no. }\end{array}$} & \multirow[b]{2}{*}{ Phage } & \multirow{2}{*}{$\begin{array}{l}\text { Multiplicity of } \\
\text { input (p.f.u.) }\end{array}$} & \multirow[b]{2}{*}{ Recipient } & \multicolumn{2}{|c|}{ Iransduction irequency/p.t.u. adsorbed } \\
\hline & & & & Kanamycin resistance & Prototrophy \\
\hline I & $5006 \mathrm{MHFT} k$ & I0 & PVI27 & $5 \times 10^{-8}$ & - \\
\hline 2 & $5006 \mathrm{MHFT} k$ & 0.6 & PVI27 & $2 \times \mathrm{IO}^{-8}$ & - \\
\hline 3 & $5006 \mathrm{MHFT} k$ & 0.6 & PM5006 & $5 \times 10^{-2}$ & - \\
\hline 4 & $5006 \mathrm{MHFT} k$ & 0.6 & PM5006arg-I & - & $I \times 10^{-8}$ \\
\hline 5 & $\begin{array}{c}\text { 5006MHFT } k \\
+ \\
5006 \mathrm{M}\end{array}$ & $\begin{array}{l}10 \\
20\end{array}$ & PVI27 & $6 \times 10^{-7}$ & - \\
\hline 6 & $\begin{array}{c}\text { 5006MHFT } k \\
+ \\
5006 \mathrm{M}\end{array}$ & $\begin{array}{l}0.6 \\
20\end{array}$ & PVI27 & $5 \times 10^{-7}$ & - \\
\hline 7 & $\begin{array}{c}5006 \mathrm{MHFT} k \\
+ \\
5006 \mathrm{M}\end{array}$ & $\begin{array}{l}10 \\
20\end{array}$ & PVI 27arg-I & $5 \times 10^{-7}$ & $<5 \times 10^{-9}$ \\
\hline 8 & $\begin{array}{c}\text { 5006MHFT } k \\
+ \\
5006 \mathrm{M}\end{array}$ & $\begin{array}{l}10 \\
20\end{array}$ & PVI27met-I & $5 \times 10^{-7}$ & $<5 \times 10^{-9}$ \\
\hline 9 & $\begin{array}{c}5006 \mathrm{MHFT} k \\
+ \\
5006 \mathrm{M}\end{array}$ & $\begin{array}{l}10 \\
20\end{array}$ & PV127ura-I & $5 \times 10^{-8}$ & $<5 \times 10^{-9}$ \\
\hline
\end{tabular}

Mobilization of possible non-self-transmissible plasmids. This was done according to Datta \& Hedges (I972), using the plasmid P-lac and also the fused plasmid P-lac $\overline{\mathbf{R} 447 \mathrm{~b}}$, and with $E$. coli strain $\mathrm{J} 62-\mathrm{I}$ as recipient on nalidixic acid-containing media.

\section{RESULTS}

Only Proteus vulgaris strain PVI27 yielded kanamycin-resistant transductants with the HFT phages. No other transductants (abortive or complete) were detected for the remaining 276 strains. Strain PVI27 was a typical $P$. vulgaris and possessed all the features which distinguish it from $P$. mirabilis strain PM5006. When the above-mentioned strains were matched on nutrient agar a demarcation line formed between their swarms (Dienes, 1946, 1947). Strain PVI 27 is sensitive to $3 \mu \mathrm{g}$ kanamycin or streptomycin $/ \mathrm{ml}$, but resistant to $100 \mu \mathrm{g}$ ampicillin $/ \mathrm{ml}$. Repeated attempts to isolate ampicillin-sensitive mutants failed but a number of auxotrophs were obtained. The ampicillin-resistance marker could not be transferred to E. coli strain J62-I directly or with the use of P-lac or P-lac $\overline{\mathrm{R} 447 \mathrm{~b}}$ as possible effectors (Datta \& Hedges, 1972), and this resistance was presumably chromosomally located. Although both HFT phages were used in further work on PVI27, results were similar and only those employing phage 5006MHFT $k$ will be described.

Plaque formation and adsorption of phages 5006M and 5006MHFTk to strain PVI 27

Neither phage formed plaques or had any visible action on PVI27. Stationary-phase and exponentially growing cultures were employed. Both the overlay technique and spotting 


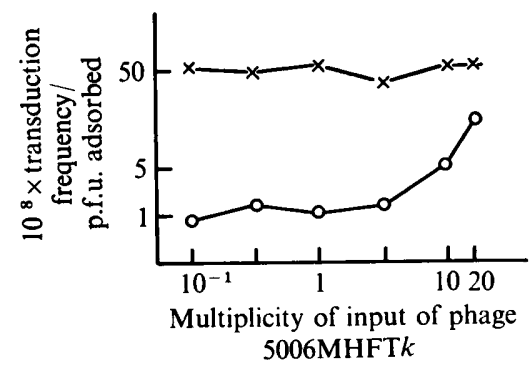

Fig. I

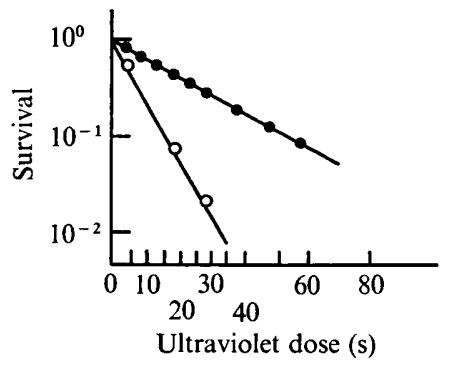

Fig. 2

Fig. I. Effect of helper phage on transduction frequency to $P$. vulgaris PVI27. $O$, Phage $5006 \mathrm{MHFT} k$ only; $\times$, phage $5006 \mathrm{MHFT} k$ with helper. Dilutions of phage $5006 \mathrm{MHFT} k$ were made in a constant concentration of phage $5006 \mathrm{M} \cdot \mathrm{PM} 5006 \mathrm{str}-\mathrm{r}$. The m.i. of this non-kanamycin resistance transducing phage was 20 p.f.u.

Fig. 2. The effect of u.v. irradiation on phage 5006MHFT $k$. Quantitative transduction experiments were done at a m.i. of I p.f.u. of unirradiated phage. A type I-S segregant of $P$. vulgaris was the recipient. $\bigcirc$, Plaque formation on $\boldsymbol{P}$. mirabilis PM5006; $\boldsymbol{O}$, transducing activity.

phage dilutions on surface lawns of the organism were investigated. Results of adsorption experiments varied (for no apparent reason) but no more than $20 \%$ of input p.f.u. were adsorbed in $20 \mathrm{~min}$. More than $99 \%$ adsorbed to PM5006 in the same time. Adsorption of phage tended to be maximal with organisms growing exponentially at $37^{\circ} \mathrm{C}$. Transduction frequencies were calculated on the assumption that $20 \%$ of input p.f.u. adsorbed to PVI 27 . In both the above aspects the phage resembled phage $\mathrm{P}_{I} \mathrm{CM}$, the chloramphenicol-resistanceconverting variant of PI (Kondo \& Mitsuhashi, 1964, 1966). This coliphage adsorbed slowly to Salmonella typhi which it lysogenized steadily, but it never formed plaques on this heterologous host. Phage 5006MHFT $k$ had no visible action on any of the 5000 clones of strain PVI 27 which had been treated with nitrosoguanidine.

\section{Transduction of PVI 27 by phage 5006MHFTk}

Expts I to 3 (Table 2) show that kanamycin-resistant transductants of PVI 27 arose at a frequency of about $5 \times 10^{-8} /$ p.f.u. adsorbed, compared with $5 \times 10^{-2} /$ p.f.u. adsorbed with PM5006 as recipient. Phage 5006M at a m.i. of about 20 p.f.u. had a helper effect (Expts 5 and 6 , Table 2) in that the transduction frequency to PVI27 was increased about tenfold. The plot (Fig. I) of transduction frequency versus m.i. in the presence and absence of phage $5006 \mathrm{M} \cdot \mathrm{PM} 5006$ str- $r$ at a constant m.i. of p.f.u. of 20 , could mean that transducing particles were defective in the sense that the transduction frequency could be increased by the presence of the latter phage, but that, because of the rapid fall of the slope between m.i. of 10 and 5 p.f.u., it was unlikely that the transducing particles complemented one another (see Chan et al. 1972; Coetzee, 1974b). The high m.i. of helper phage required was possibly the result of poor adsorption, as a m.o.i. of 3 was sufficient for homologous transductions (Coetzee, I974 b, I975; see also Expts 3 and I0, Table 3). The possibility that phage $5006 \mathrm{M}$ was merely causing a 'multiplicity effect' (see Heip, Rolfe \& Schell, 1974) could not be ruled out, but is unlikely because a host-controlled modification system does not appear operative in these particular transductions (see below). Resistance to $50 \mu \mathrm{g}$ kanamycin/ml was complete immediately after the $20 \mathrm{~min}$ adsorption, but resistance to $500 \mu \mathrm{g} / \mathrm{ml}$ was only achieved after membranes with impinged bacteria had been incubated on non-selective media for $\mathrm{I} h$. This is similar to the transduction of kanamycin resistance to PM5006 by this phage (Coetzee, I974b). Phage 5006MHFT $k$ is capable of generalized 
transduction of PM5006 at low frequencies (Expt 4, Table 2) but the phage was not able to transduce auxotrophs of PVI27 to phototrophy (Expts 7 to 9, Table 2).

Minute colonies possibly indicative of abortive transductants were never observed. It was thought that the $\mathrm{I} h$ pre-incubation of membranes on nutrient agar could be responsible, by allowing more time for chromosomal integration; therefore some experiments were repeated in which membranes were placed on to kanamycin-MacConkey agar directly after filtration, but minute colonies were not observed.

\section{Properties of PVI 27 kanamycin-resistant transductants}

Transductants retained all the distinguishing features of $P$. vulgaris and could be subdivided on the grounds of the m.i. of the transducing phage. Those produced at low m.i. $(<\mathrm{I})$ of p.f.u. (type I) did not liberate phage, either spontaneously or on u.v. induction, which could plate on PM5006. Chances of re-infection of these transductants were slight because of the low adsorption rate of the phage and the phage 34 antiserum used in critical experiments. These type I transductants presumably resulted from single-particle infection, and this would indicate that transducing particles were able to lysogenize on single infection. They were, however, defective in some maturation function, for although these transductants yielded many kanamycin-sensitive segregants, named I-S (see below), no phage active on PM5006 was liberated. These transductants still formed a Dienes demarcation line when their swarms were matched against those of PM5006, and adsorbed the phages to about the same extent as PVI27. Phage $5006 \mathrm{M}$ or $5006 \mathrm{MHFT} k$ (titre $5 \times 10^{9} \mathrm{p.f.u} . / \mathrm{ml}$ ) when spotted on lawns of these transductants formed turbid areas surrounded by razor-thin lines of clearing. Upon I0- or 20-fold dilution of the phage this effect disappeared. Purified clones from these circumscribed areas yielded phage spontaneously and on u.v. induction. The presence of the transducing phage genome produced by single-particle infection appeared to be essential for the 'circumscribed area' phenomenon-it was not observed with PVI 27, I-S segregants or any other PVI 27 derivatives. No ready explanation for the phenomenon is available: while phage $5006 \mathrm{M}$ exerted a helper effect on transduction frequency (and thus presumably on lysogenization) it was unlikely (see below) that particles of phage 5006MHFT $k$ assisted one another in this respect although another form of complementation between these particles possibly exists (see Discussion). Transductants which resulted from a phage m.i. of $>$ Io (type II) liberated phage spontaneously and titres of $2 \times 10^{9}$ p.f.u./ml on PM5006 could be obtained by u.v. induction. They failed to adsorb phages $5006 \mathrm{M}$ or $5006 \mathrm{MHFT} k$.

A striking feature of all transductants, and in marked contrast to the stable chloramphenicol-resistant lysogens which phage PICM produced in $S$. typhi (Kondo \& Mitsuhashi, I966), was the rate at which PVI 27 transductants lost the kanamycin resistance. After overnight growth in broth, 5 to I $5 \%$ of colony-forming units were sensitive to $3 \mu \mathrm{g}$ kanamycin/ $\mathrm{ml}$. This high segregation rate from all transductants favours the view that transduction was by addition, i.e. lysogenization (Luria, Adams \& Ting, 1960; Hoppe \& Roth, 1974) rather than substitution as a result of a double cross-over event. Another explanation of the high segregation rate was that the phages functioned as plasmids whose replication was not perfectly attuned to the division mechanism of PVI27 (see Signer, 1969). Lack of genetic knowledge concerning the phages prevented further investigation of this aspect. One hundred and fifty-four type I-S segregants were investigated. They originated from the same number of type I transductants (I5 individual experiments) which did not liberate phage. These segregants were non-lysogenic, in the sense that they too did not liberate phage, and adsorbed some phage, but with no greater efficiency than the corresponding transductants 
Table 3. Transduction by u.v.-induced lysates derived from a kanamycin-resistant P.vulgaris PVI 27 transductant

\begin{tabular}{|c|c|c|c|c|c|}
\hline \multirow[b]{2}{*}{$\begin{array}{l}\text { Experiment } \\
\text { no. }\end{array}$} & \multirow[b]{2}{*}{ Lysate } & \multirow{2}{*}{$\begin{array}{l}\text { Multiplicity } \\
\text { of } \\
\text { input } \\
\text { (p.f.u.) }\end{array}$} & \multirow[b]{2}{*}{ Recipient } & \multicolumn{2}{|c|}{$\begin{array}{l}\text { Transduction frequency/p.f.u. } \\
\text { adsorbed }{ }^{*}\end{array}$} \\
\hline & & & & $\begin{array}{c}\text { Kanamycin } \\
\text { resistance }\end{array}$ & Prototrophy \\
\hline I & u.v.-II & I0 & PVI 27 & $4 \times 10^{-8}$ & - \\
\hline 2 & u.v.-II & 0.7 & PVI 27 & $4 \times 10^{-8}$ & - \\
\hline 3 & u.v.-II & 0.6 & PM5006 & $6 \times 10^{-2}$ & - \\
\hline 4 & u.v.-II & 0.6 & Segregant I-S & $5 \times 10^{-8}$ & - \\
\hline 5 & u.v.-II & 0.6 & Segregant II-Sa & $<5 \times 10^{-9}$ & - \\
\hline 6 & u.v.-II & 0.5 & Sergegant II-Sb & $8 \times 10^{-7}$ & - \\
\hline 7 & u.v.-II & 0.5 & Segregant II-Sc & $8 \times 10^{-7}$ & - \\
\hline 8 & $\begin{array}{l}\text { u.v.-II } \\
+ \\
5006 \mathrm{M}\end{array}$ & $\begin{array}{l}0.7 \\
20\end{array}$ & Segregant II-Sc & $6 \times 10^{-6}$ & - \\
\hline 9 & $\begin{array}{c}\text { u.v.-II } \\
+ \\
5006 \mathrm{M}\end{array}$ & $\begin{array}{l}0.5 \\
20\end{array}$ & PVI 27argn $I$ & $5 \times 10^{-7}$ & $<5 \times 10^{-9}$ \\
\hline Io & $\begin{array}{c}\text { u.v.-II } \\
+ \\
\text { 5006M·PM5006arg-I }\end{array}$ & $\begin{array}{l}0 \cdot 7 \\
3\end{array}$ & PM5006arg-I & $3 \times 10^{-1}$ & $<\mathrm{I} \times 10^{-9}$ \\
\hline I I & $\begin{array}{c}\text { u.v.-II } \\
+ \\
\text { 5006M·PM5006lys-I }\end{array}$ & $\begin{array}{l}1 \\
20\end{array}$ & PM5006lys- $r$ & $1 \times 10^{-1}$ & $<\mathrm{I} \times \mathrm{IO}^{-9}$ \\
\hline 12 & $\begin{array}{c}\text { U.v.-II } \\
+ \\
\text { 5006M·PM5006trp-I }\end{array}$ & $\begin{array}{r}5 \\
10\end{array}$ & PM5006trp-I & $I \times 10^{-1}$ & $<\mathrm{I} \times \mathrm{IO}^{-9}$ \\
\hline
\end{tabular}

Transductions were done as outlined in Methods and the legend to Table 2.

* Calculated for PVI27 and derivatives as $20 \%$ of m.i.

or PVI27. Seventy-nine segregants which arose from phage-liberating transductants (type II) were also studied. These were of three types. Supernatants of overnight broth cultures of the 49 type II-Sa segregants contained phage which plated on PM5006. These segregants did not adsorb phages $5006 \mathrm{M}$ or $5006 \mathrm{MHFT} k$. The 22 type II-Sb segregants only liberated phage on u.v. induction, while phage liberation from the eight type II-Sc segregants could not be demonstrated. Segregant types II-Sb and II-Sc adsorbed about $20 \%$ of phage 5006MHFT $k$ in $20 \mathrm{~min}$. Neither phage $5006 \mathrm{MHFT} k$ nor phage $5006 \mathrm{M}$ was ever observed to form plaques on any segregant.

The phage adsorption experiments showed that the low kanamycin-resistance transduction frequency of PVI27 could not be explained on the grounds of host-range mutants of PVI 27 capable of phage adsorption (Kondo \& Mitsuhashi, I966).

Phage 5006M·PM5006str-r could not (results not shown) transduce streptomycin resistance to PVI27, to kanamycin-resistant transductants of type I or to segregant types I-S, II-Sb and II-Sc. Small doses of u.v. irradiation to the phage lysate (Coetzee \& Sacks, I960) did not affect this.

\section{Properties of phage derived from transductants}

A type II PVI27 transductant was induced by u.v. irradiation. The lysate had a titre of $2 \times 10^{9}$ p.f.u./ml on PM5006, to which more than $99 \%$ of the phage adsorbed within 10 min. The phage was neutralized at the same rate $(K=170)$ as phage $5006 \mathrm{M}$ by antiserum to 
phage 34. It did not form plaques on PVI27 type I transductants or any of the segregants, but adsorbed with similar low frequencies as phage $5006 \mathrm{M}$ to strains which adsorbed the latter phage. Lysates of other type II transductants had similar properties, and it was concluded that transduction of PVI 27 by phage 5006MHFT $k$ was not due to a mutant of the latter phage with an increased rate of adsorption to the $P$. vulgaris strain. This lysate (and lysates of other type II transductants, not shown) could transduce PVI27, segregant types I-S, II-Sb, II-Sc and PM5006 to kanamycin resistance (Expts I to 4, 6 and 7, Table 3). The transduction frequencies of PVI27 were similar to those employing phage 5006MHFTk (compare Expts I and 2, Table 2) and ruled out host-controlled modification as the cause of the low transduction frequency. Kondo \& Mitsuhashi(1966) also ruled out host-controlled modification in their E. coli-S. typhi PICM transduction system on similar grounds. Using segregants of type II-Sa as recipients (Expt 5, Table 3 ) no transductants were obtained. The phage did not adsorb to these segregants (see above). Transduction frequencies to segregant types II-Sb and II-Sc (Expts 6 and 7, Table 3) were invariably greater than to PVI 27 (Expts I and 2, Table 3). Again no abortive transductants were observed. Provided the phage present in type II-Sb segregants had a functioning immunity system this was the exact situation where abortive transductants should have been encountered (Ozeki \& Ikeda, I968). These transductants of segregants lost the kanamycin-resistance marker at the same high rate as PVI27 transductants. The segregants could be re-transduced at the same frequencies as the original segregants. Transduction frequencies were increased about tenfold by the presence of phage $5006 \mathrm{M}$ at a m.i. of about 20 p.f.u. (compare Expts 7 and 8 , Table 3). Lysates derived from type II transductants transduced PM5006 to kanamycin resistance at the same frequencies as phage 5006MHFT $k$ (see Expt 3, Table 2 and Expt 3, Table 3 ) but the general transducing properties of the latter phage (Coetzee, 1974 $b$ ) were not evident even with the use of helper non-marker transducing phage raised on the recipient (Expts 9 to I2, Table 3). The helper phage did increase the frequency of the corresponding kanamycin-resistance transductions (compare Expts 3 and I0, Table 3). All PM5006 kanamycin-resistant transductants retained the biochemical reactions of $\boldsymbol{P}$. mirabilis. Just as with phage $5006 \mathrm{MHFT} k$ (Coetzee, $1974 b$ ), transductants of PM5006 by phage derived from type II PVI 27 transductants at m.o.i. of $<0 \cdot$ I were lysogenic and failed to adsorb the phages. This could be explained by the fact that PM5006 harboured a cryptic prophage (Krizsanovich, I 973, see Coetzee, I974 $b$ ). Ultraviolet-induced lysates of these transductants (which also yielded kanamycin-sensitive segregants at the same high rate) yielded phage with properties similar to phage 5006MHFT $k$. With the use of phage 5006MHFTak to transduce PVI27 to kanamycin resistance, the phage which emerged from transductants transduced PM5006 to ampicillin and kanamycin resistance although no selection for ampicillin resistance was applied to PVI27 (results not shown). None of the induced phages produced abortive transductants.

\section{Ultraviolet irradiation of transducing lysates}

Plots of time of irradiation of various HFT lysates and kanamycin-resistance transduction frequencies, employing PVI27 and a number of kanamycin-sensitive segregants of PVI27 transductants as recipients, revealed simple exponential declines of transduction frequency as a function of time of irradiation. The example presented in Fig. 2 confirmed the conclusion reached from the high segregation rates of the kanamycin-resistance marker, that transduction was by lysogenization (either as prophage or plasmid) and not by double cross-over events between marker and the PVI 27 chromosome (Luria et al. 1960; Coetzee, I $974 b$ ). 


\section{DISCUSSION}

Results of experiments reported here indicate that transduction by the HFT $P$. mirabilis phages was almost entirely limited to the original host. The single exception $-P$. vulgaris strain PVI 27 - adsorbed the phages very poorly. Even this could hardly have been detected without the use of a high-frequency antibiotic-resistance transducing phage, and some features of the system merit attention.

The method used for selection of a mutant host capable of greater phage adsorption and consequent plaque formation, was not successful (see Goldberg et al. 1974), and phage adsorption tests on many transductants of PVI27 and segregant clones also did not yield any with a greater affinity than PVI27 itself. The nature of the transduction process which occurred here was established as analogous to heterogenote formation and was to be expected in conditions where possibly too little microhomology existed for double cross-over events to occur (see Coetzee, 1972; Luria et al. 1960).

That transductants, formed at low multiplicities of phage input (type I transductants), adsorbed homologous phage is unique for these phages. Transductants of their host, PM5006, did not adsorb the phages. This was attributed to lysogenic conversion (Coetzee, 1961). Strain PM5006 is cryptically lysogenic for an homologous prophage (Krizsanovich, 1973) and complementation of genes of transducing particles by those of the prophage hampered assessment of possible defectiveness of the transducing phages (Coetzee, 1974 $b$ ). Type I transductants could result from single-particle transduction by particles defective for the converting gene and defective in some maturation function, for although transductants segregated kanamycin-sensitive clones at a high rate they did not release viable phage. The fact that type II transductants did not adsorb the phages could indicate complementation of this non-adsorption function by another prophage. This could indicate a certain heterogeneity in the population of transducing particles not previously detected. However, there was no means available for testing the effect of single-particle infection by wild-type phage on PVI27, and the problem remains unsolved.

Phage-adsorbing segregant types II-Sb or II-Sc, derived from non-phage-adsorbing transductants, may have arisen by permutations of the prophage excision event producing gene rearrangements which resulted in faulty phage-adsorption gene products. As phage lysates of type II PVI27 kanamycin-resistant transductants transduced PVI27 to kanamycin resistance at the same low frequency as phage 5006MHFTk, and transduced PM5006 to this resistance at the same frequency as the latter phage, host cell-modification systems did not appear to play a role in the discrepancy demonstrated. However, despite good adsorption to PM5006, the phage which originated from PVI27 did not possess the general transducing properties of the parent phage 5006MHFTk. Strain PM5006 possesses a hostcontrolled modification system (Coetzee \& Smit, 1970). It may well be that chromosomal material carried by the generalized transducing fraction of the HFT lysate was restricted by this system, but that material coding for kanamycin (or ampicillin) resistance escaped restriction because of its different ( $R$ plasmid) origin (see Inselburg, 1966). The host-controlled modification status of PVI 27 is not known, but failure to detect streptomycinresistant transductants of PVI27 by phage 5006M-PM5006str-r, and also the failure of the generalized transduction potential of phage 5006MHFT $k$ to register, could mean that here again generalized transduction markers were restricted. However, this does not always apply because Coetzee \& Smit (I969) found that generalized transducing phage $34^{\circ}$ PMI 3 transduced markers of strain PMI 3 to a recipient, which severely restricted the phage, at a frequency one-tenth of that to strain PMI3. While poor particle adsorption coupled to the 
low frequency of generalized transduction (Coetzee, 1974 $b$ ) could play a role, other factors, such as differences in composition of HFT and generalized transducing particles (Ozeki \& Ikeda, 1968; see also Holloway, Krishnapillai \& Stanisich, 197I) and mode of integration of the particles into the bacterial chromosome (Luria et al. 1960), could be important.

A surprising feature was the absence of abortive transductants in attempts at generalized, as well as kanamycin-resistance transductions. These were systems where, because of possible lack of homology, abortive transduction would be expected to occur. Demerec \& Ohta (1964) found many abortive transductants in P22 transductions between $S$. typhimurium and E. coli, and Chakrabarty \& Gunsalus (1970) encountered them in transductions between Pseudomonas aeruginosa and Ps. putida. The workers mentioned above employed general transducing phage. Failure to demonstrate generalized transduction may be accounted for by the poor adsorption and the low frequency of this process (see above). There is, however, no obvious reason why HFT defective phages that are also defective in circularization or chromosome attachment function should not produce abortive transductants (Ozeki \& Ikeda, I968). It must be assumed that transducing lysates did not contain particles of this kind.

A consistent finding was that segregant types II-Sb and II-Sc were transduced to kanamycin resistance at higher frequencies than type I-S or PVI27. Segregation rates, as well as the effect u.v. irradiation of transducing lysates had on transduction frequencies, indicated that transduction proceeded by lysogenization. It is suggested that these segregants still possessed phage genetic material which provided better (or more) phage attachment sites than type I-S or PVI 27 had at their disposal. This (presumed) phage resident material, like cryptic prophage $5006 \mathrm{M}$, could not substitute for the helper effect of vegetative phage, the function of which remains obscure (Coetzee, 1974b). However, the fact that homologous non-transducing phage exerted a helper effect in transductions to both $P$. mirabilis PM5006 and $P$. vulgaris PVI27 emphasized that the primary defect was with the phage and not its hosts.

Observations (not shown) which indicated that phages 5006MHFT $k$ and 5006MHFTak behaved similarly with regard to PVI27 meant that the reactions studied were not subtle enough to detect differences or that the additional marker of phage 5006MHFTak exerted no other phenotypic effect on the phage (see Coetzee, 1975).

The author is grateful for cultures provided by Drs Naomi Datta, H. C. de Klerk, R. W. Hedges and H. J. Koornhof. This work was aided by grants from the South African Medical Research Council.

\section{REFERENCES}

Adams, M. H. (1956). Methods of study of bacterial viruses. Methods in Medical Research 2, I-73.

Adelberg, E. A., Mandel, M. \& Chen, G. C. C. (I965). Optimal conditions for mutagenesis by $N$-methyl$N^{\prime}$-nitro- $N$-nitrosoguanidine in Escherichia coli KI2. Biochemical and Biophysical Research Communications 18, 788-795.

Brenner, D. J. \& FAlKow, S. (1971). Molecular relationships among members of the Enterobacteriaceae. Advances in Genetics 16, 81-1 18 .

CARPenter, K. P. (1964). The Proteus-Providence group. In Recent advances in Clinical Pathology, series IV, pp. 13-24. London: Churchill.

Chakrabarty, A. M. \& Gunsalus, I. C. (I970). Transduction and genetic homology between Pseudomonas species putida and aeruginosa. Journal of Bacteriology 103, 830-832.

Chan, R. K., Botstein, D., Watanabe, T. \& Ogata, Y. (1972). Specialized transduction of tetracycline resistance by phage $\mathrm{P}_{22}$ in Salmonella typhimurium. Virology 50, 883-898.

Clowes, R. C. \& HAYes, W. (1968). Experiments in Microbial Genetics. Oxford and Edinburgh: Blackwell Scientific Publications. 
Coetzee, J. N. (I96I). Lysogenic conversion in the genus Proteus. Nature, London r89, 946-947.

CoetzeE, J. N. (I963 a). Lysogeny in Proteus rettgeri and the host-range of $P$. rettgeri and $P$. hauseri bacteriophages. Journal of General Microbiology 31, 219-229.

Coetzee, J. N. (1963 b). Lysogeny in Providence strains and the host-range of Providence bacteriophages. Nature, London 197, 515-5I6.

Coetzee, J. N. (1963c). Host-range of Proteus morganii bacteriophages. Nature, London 199, 827-828.

Coetzee, J. N. (1972). Genetics of the Proteus group. Annual Review of Microbiology 26, 23-54.

Coetzee, J. N. (1974a). Properties of Proteus and Providence strains harbouring recombinant plasmids between P-lac and RIdrd 19 or R447b. Journal of General Microbiology 80, I I9-130.

Coetzee, J. N. (1974b). High frequency transduction of kanamycin resistance in Proteus mirabilis. Journal of General Microbiology 84, 285-296.

CoetzeE, J. N. (1975). High frequency transduction of resistance to ampicillin and kanamycin in Proteus mirabilis. Journal of General Microbiology 87, I73-176.

Coetzee, J. N., Datta, N. \& Hedges, R. W. (1972). R factors from Proteus rettgeri. Journal of General Microbiology 72, 543-552.

CoetzeE, J. N. \& SACKs, T. G. (1960). Transduction of streptomycin resistance in Proteus mirabilis. Journal of General Microbiology 23, 445-455.

Coetzee, J. N. \& Smit, J. A. (1969). Restriction of a transducing bacteriophage in a strain of Proteus mirabilis. Journal of General Virology 4, 593-607.

Coetzee, J. N. \& Smit, J. A. (1970). Properties of Proteus mirabilis phage 13vir. Journal of General Virology 9, 247-249.

Coetzee, J. N., Smit, J. A. \& Prozesky, O. W. (1966). Properties of Providence and Proteus morganii

transducing phages. Journal of General Microbiology 44, 167-176.

Datta, N. \& Hedges, R. W. (1972). Host ranges of R factors. Journal of General Microbiology 70, 453-460.

Demerec, M. \& OHTA, N. (1964). Genetic analyses of Salmonella typhimurium Escherichia coli hybrids. Proceedings of the National Academy of Sciences of the United States of America 52, 317-323.

Dienes, L. (1946). Reproductive processes in Proteus cultures. Proceedings of the Society for Experimental Biology and Medicine 63, 265-270.

DIENES, L. (I 947). Further observations on the reproduction of bacilli from large bodies in Proteus cultures. Proceedings of the Society for Experimental Biology and Medicine 66, 97-98.

Falkow, S., Ryman, I. R. \& Washington, O. (1962). Deoxyribonucleic acid base composition of Proteus and Providence organisms. Journal of Bacteriology 53, I3 $18-132 \mathrm{I}$.

Falkow, S., Wohlhieter, J. A., Citarella, R. V. \& Baron, L. S. (I964). Transfer of episomic elements to Proteus. Journal of Bacteriology 88, I598-160I.

Goldberg, R. B., Bender, R. A. \& Streicher, S. L. (1974). Direct selection for PI-sensitive mutants of enteric bacteria. Journal of Bacteriology I $18,8 \mathrm{IO}-8 \mathrm{I} 4$.

Gottesman, M. M., Hicks, M. L. \& Gellert, M. (I973). Genetics and functions of DNA ligase in Escherichia coli. Journal of Molecular Biology 77, 53 I-547.

Hedges, R. W., Datta, N., Coetzee, J. N. \& Dennison, S. (1973). R factors from Proteus morganii. Journal of General Microbiology 77, 249-259.

Heip, J., Rolfe, B. \& Schell, J. (1974). Abolition of host cell restriction by high multiplicity of phage infection. Virology 59, 356-370.

Holloway, B. W., Krishnapillai, V. \& Stanisich, V. (1971). Pseudomonas genetics. Annual review of Genetics 5, 425-446.

Hoppe, I. \& Roth, J. (1974). Specialized transducing phages derived from Salmonella phage P22. Genetics $76,633-654$.

InSElburg, J. (1966). Phage PI modification of bacterial DNA studied by generalized transduction. Virology 30, $257-265$.

Kondo, E. \& MitsuHAShi, S. (I964). Drug resistance of enteric bacteria. IV. Active transducing bacteriophage PICM produced by the combination of R factor with bacteriophage PI. Journal of Bacteriology 88, $1266-1276$.

Kondo, E. \& Mitsuhashi, S. (1966). Drug resistance of enteric bacteria. VI. Introduction of bacteriophage PICM into Salmonella typhi and formation of PIdCM and F-CM elements. Journal of Bacteriology 9r, I $787-1794$.

Krizsanovich, K. (1973). Cryptic lysogeny in Proteus mirabilis. Journal of General Virology r9, 3 I I-320. Luria, S. E., Adams, J. N. \& Ting, R. C. (1960). Transduction of lactose-utilizing ability among strains of $E$. coli and $S$. dysenteriae and the properties of the transducing phage particles. Virology 12, 348-390. Ozeki, H. \& IKedA, H. (I968). Transduction mechanisms. Annual Review of Genetics 2, 245-278.

Signer, E. R. (1969). Plasmid formation: a new mode of lysogeny by phage $\lambda$. Nature, London 223, I $58-160$ 